\title{
Reply to comment on Sen et al.: Osteosynthesis of femoral-neck nonunion with angled blade plate and autogenous fibular graft
}

\author{
Sujit Kumar Tripathy • Ramesh Kumar Sen • \\ Tarun Goyal
}

Received: 8 January 2012 / Accepted: 9 January 2012 / Published online: 26 January 2012

(C) Springer-Verlag 2012

The queries of the author raised to our published article [1] have been answered below point by point.

1. Valgus intertrochanteric osteotomy may be considered in femoral neck nonunion with varus malalignment. It converts the vertical shear force into a horizontal compressive force and promotes union. However, it alters the biomechanics of the hip joint and causes shortening, limp and a decreased range of movement, probably because of increased pressure on the femoral head leading to degenerative disease or osteonecrosis $[1,2]$. Considering the long-term problems of osteotomy, Roshan et al. suggested that bone grafting with internal fixation is still the reliable method of fixation in femur neck nonunion [2]. The patients with femoral neck nonunion in our series had no gross varus malalignment $\left(>110^{\circ}\right)$. We recommend fibular graft fixation with angle stable device in those cases where there is no or minimal varus malalignment (neck shaft angle $>110^{\circ}$ ). Patients with significant varus malalignment can be managed by valgus osteotomy with angled blade plate fixation with or without fibular graft.

2. Static compression at the fracture site can be achieved with parallel screws, but dynamic compression in the

S. K. Tripathy $\cdot$ R. K. Sen $\cdot$ T. Goyal

Postgraduate Institute of Medical Education and Research, Chandigarh 160012, India

S. K. Tripathy $(\bowtie)$

Department of Orthopaedics, Friarage Hospital,

Northallerton, United Kingdom DL6 1JG

e-mail: sujitortho@yahoo.co.in

T. Goyal

Freeman Hospital,

Newcastle, UK postoperative period is not advisable in cases of nonunion management when fibular graft is used to augment osteogenesis. Early weight bearing to achieve dynamic compression at the fracture site may lead to implant failure (screws back out from the lateral cortex) and shear force on the fibular graft. Because of variable resorption at the fracture site in femur neck nonunion, the surface area of contact is less in comparison to a fresh fracture. By opening the fracture site and even freshening the bone surfaces on both sides, the bone apposition may not be completely anatomical, hence near anatomical reduction is achieved in most nonunions. This makes the fracture site more unstable. Three main deforming forces act at the fracture site: bending force, rotational torsion and axial compressive force (beneficial, but unfortunately not acceptable in femoral neck nonunion where cannulated screws are used along with the fibular graft). It is well proven in literature that angled blade plate (rectangular shape) provides better resistance to these deforming forces compared to cannulated screws [3]. In mathematical calculations also, the surface contact area of angled blade plate is more than two screws. The angled blade plate traverses through multiple trabeculae to engage in the femoral head.

3. The blade plates are rectangular in shape with a groove on the upper side (U profile) providing rigid stability and preventing rotational torsion. The longer plate of an angled blade plate may contribute to wider load distribution [3].

4. We admit that a blade plate has the potential complication of hip joint penetration and cut out from the superior surface. This can be avoided by appropriate placement (middle or inferior half of femur head and neck) of the blade plate and total circumferential 
evaluation of the hip joint by the image intensifier intraoperatively. Hip joint penetration and screw cut-out have also been documented with cannulated cancellous screw fixation.

5. The previous fixation tracts do not cause any problem in angled blade plate fixation, because the previous implant tracts are circular and the blade plate is rectangular. The chisel for the angled blade plate can be hammered over the previous tract. The technique is challenging and is highly dependent on the intraoperative image intensifier. This procedure can be performed by an average orthopaedic surgeon provided an image intensifier facility is available. Femoral neck dimensions are adequate for placement of a fibular graft and an angled blade plate; there may not be adequate room for additional screw fixation.

6. The precautions in angled blade plate fixation for fractured neck femur have been described by Raaymakers and Marti [4]. Provisional K-wire fixation and drilling the tract with a $4.5-\mathrm{mm}$ drill bit before hammering with chisel avoids the risks of micro-fracture and distraction of the fracture fragments. The chisel should be hammered a little bit further than (at least $5 \mathrm{~mm}$ ) the expected blade plate length.

An appropriately fixed angled blade plate would be definitely superior to cannulated screws fixation in terms of mechanical stability when used along with the fibular graft in femoral neck nonunion $[1,5]$.

Conflict of interest disclosure None

Support None

\section{References}

1. Sen RK, Tripathy SK, Goyal T, Aggarwal S, Tahasildar N et al. (2011) Osteosynthesis of femoral-neck nonunion with angled blade plate and autogenous fibular graft. Int Orthop. Sep 1. [Epub ahead of print]. doi:10.1007/s00264-011-1344-1

2. Roshan A, Ram S (2008) The neglected femoral neck fracture in young adults: review of a challenging problem. Clin Med Res 6 (1):33-39

3. Mataliotakis G, Lykissas MG, Mavrodontidis AG, Kontogeorgakos VA, Beris AE (2009) Femoral neck fractures secondary to renal osteodystrophy. Literature review and treatment algorithm. J Musculoskelet Neuronal Interact 9(3):130-137

4. Raaymakers EL, Marti RK (2008) Nonunion of the femoral neck: Possibilities and limitations of the various treatment modalities. Indian J Orthop 42:13-21

5. Tripathy SK, Goyal T, Sen RK (2011) Revision internal fixation and nonvascular fibular graft for femoral neck nonunion. J Trauma 71 (1):270-271 